\title{
Epidemiology and predictors of traumatic spine injury in severely injured patients: implications for emergency procedures
}

\author{
David Häske $^{1,2}$ D $\cdot$ Rolf Lefering ${ }^{3} \cdot$ Jan-Philipp Stock ${ }^{4} \cdot$ Michael Kreinest $^{5} \cdot$ The TraumaRegister DGU $^{6}$
}

Received: 29 July 2020 / Accepted: 25 September 2020 / Published online: 6 October 2020

(c) The Author(s) 2020, corrected publication 2022

\begin{abstract}
Purpose This study aimed to identify the prevalence and predictors of spinal injuries that are suitable for immobilization. Methods Retrospective cohort study drawing from the multi-center database of the TraumaRegister DGU ${ }^{\circledR}$, spinal injury patients $\geq 16$ years of age who scored $\geq 3$ on the Abbreviated Injury Scale (AIS) between 2009 and 2016 were enrolled.

Results The mean age of the 145,833 patients enrolled was $52.7 \pm 21.1$ years. The hospital mortality rate was $13.9 \%$, and the mean injury severity score (ISS) was $21.8 \pm 11.8$. Seventy percent of patients had no spine injury, $25.9 \%$ scored $2-3$ on the AIS, and 4.1\% scored 4-6 on the AIS. Among patients with isolated traumatic brain injury (TBI), 26.8\% had spinal injuries with an AIS score of 4-6. Among patients with multi-system trauma and TBI, 44.7\% had spinal injuries that scored 4-6 on the AIS. Regression analysis predicted a serious spine injury (SI; AIS 3-6) with a prevalence of $10.6 \%$ and cervical spine injury (CSI; AIS 3-6) with a prevalence of 5.1\%. Blunt trauma was a predictor for SI and CSI (OR 4.066 and OR 3.640, respectively; both $p<0.001)$ and fall $>3 \mathrm{~m}$ for SI (OR 2.243; $p<0.001)$ but not CSI (OR 0.636; $p<0.001)$. Pre-hospital shock was predictive for SI and CSI (OR 1.87 and OR 2.342, respectively; both $p<0.001$ ), and diminished or absent motor response was also predictive for SI (OR 3.171) and CSI (OR 7.462; both $p<0.001)$. Patients over 65 years of age were more frequently affected by CSI.

Conclusions In addition to the clinical symptoms of pain, we identify ' $4 \mathrm{~S}$ ' [ spill (fall) $>3$ m, seniority (age $>65$ years), seriously injured, skull/traumatic brain injury] as an indication for increased attention for CSIs or indication for spinal motion restriction.
\end{abstract}

Keywords Immobilization $\cdot$ Prehospital $\cdot$ Risk $\cdot$ Trauma

\section{Abbreviations}

AIS Abbreviated Injury Scale

CSI Cervical spine injury

ECS Eppendorf-Cologne Scale

ISS Injury severity score
SCI Spinal cord injuries

TBI Traumatic brain injury
David Häske

haeske@ rettungsdienst-reutlingen.de

Rolf Lefering

rolf.lefering@uni-wh.de

Jan-Philipp Stock

jan-philipp.stock@web.de

Michael Kreinest

michael.kreinest@bgu-ludwigshafen.de

1 German Red Cross, Emergency Medical Service, Obere Wässere 1, 72764 Reutlingen, Germany

2 Center for Public Health and Health Services Research, University Hospital Tübingen, Tübingen, Germany
3 Institute for Research in Operative Medicine (IFOM), University Witten/Herdecke, Cologne, Germany

4 Department of Anesthesiology, Intensive Care Medicine, Emergency and Pain Medicine, Klinikum am Steinenberg, Reutlingen, Germany

5 Department of Trauma and Orthopedic Surgery, BG Trauma Center Ludwigshafen, Ludwigshafen, Germany

6 Committee on Emergency Medicine, Intensive Care and Trauma Management (Sektion NIS) of the German Trauma Society (DGU), Cologne, Germany 


\section{Introduction}

According to the literature, only $1-2 \%$ of all trauma patients suffer from relevant spinal injuries [1]. These patients are at risk of spinal cord injuries (SCIs) with severe neurological consequences, which occur in approximately $20 \%$ of these cases [2]. Because spinal immobilization can reduce spinal movement [3], the immobilization of patients with SCIs has been recommended since the mid-twentieth century to prevent further damage. Since then, immobilization has become a standard procedure in emergency medicine [2]; however, a recent study found that $20 \%$ of patients suffering from a cervical SCI were not immobilized whatsoever by emergency care providers [4]. A possible explanation is the fact that, over the years, no evidence for the benefit of immobilization for patient outcomes has been obtained from rigorous studies (e.g., randomized controlled trials) [5-7]. Furthermore, regardless of the method, immobilization has its disadvantages, such as general patient manipulation, pain, decubitus, and prolonged pre-hospital time $[8,9]$.

Therefore, a differentiated indication is recommended. In many cases, decision rules are established based on the circumstances of the accident (e.g., accident mechanism) and the patient's symptoms, but also from studies with healthy volunteers or corpses, whose results were generalized to trauma patients $[5,7]$. The most well-known decision-making aids for spinal immobilization are the NEXUS criteria and the Canadian C-Spine Rule (CCSR), and, in Europe, the E.M.S. IMMO protocol and the Scandinavian recommendations [10-13]. All of these decision rules include possible predictors that should detect an increased probability of spinal injury in trauma patients.

However, the decision rules were often created for the indication of imaging to establish a definitive diagnosis; they only partially evaluated for pre-hospital care [12-14]. But, for emergency procedures, however, the aim is not to make an exact diagnosis but to identify the indication for immobilization to prevent possible consequential damage during extrication and transport.

From this point of view, studies on predictors must be considered.

In an 8-year European cohort study of patients with spinal fractures, Hasler et al. showed that falls from $>3 \mathrm{~m}$ were the strongest predictor for spine trauma [15]. In addition, cervical spine injuries were most common in traffic accidents, sports, and falls $>2 \mathrm{~m}$, as well as in patients $>65$ years-old [16]. A registry study on spinal cord injuries showed that traumatic brain injuries (TBIs), shock at the scene, the severity of injuries, and age $>60$ years, worsen the outcome [17]. While anatomy has not changed over the decades, leisure behaviors and safety techniques have (e.g., wearing seatbelts in vehicles) $[18,19]$, and with it the question of the generalizability of non-European studies [12, 13, 20].

\section{Goals of this investigation}

As such, this study aims to identify the epidemiological characteristics and possible predictors of spinal injury in severely injured patients in Europe.

\section{Materials and methods}

\section{Study design}

Data from the TraumaRegister DGU ${ }^{\circledR}$ (TR-DGU) were retrospectively analyzed.

\section{Database}

The TR-DGU of the German Trauma Society [Deutsche Gesellschaft für Unfallchirurgie (DGU)] was founded in 1993. The aim of this multi-center database is to collect pseudonymized and standardized documentation of severely injured patients. Data are collected prospectively in four consecutive phases from the site of the accident until discharge from hospital: pre-hospital phase, emergency room and initial surgery phase, intensive care phase, and discharge phase. The documentation includes detailed information on demographics, injury pattern, comorbidities, pre- and inhospital management, the course in the intensive care unit (ICU), relevant laboratory findings (including data on transfusion), and the outcomes of each patient. The inclusion criteria comprised (1) hospital admission via an emergency room with subsequent ICU care; (2) vital signs upon hospital arrival; or (3) death prior to ICU admission.

The infrastructure for documentation, data management, and data analysis is provided by the Academy for Trauma Surgery (AUC; Akademie der Unfallchirurgie $\mathrm{GmbH}$ ), a company affiliated to the German Trauma Society. The scientific leadership is provided by the Committee on Emergency Medicine, Intensive Care and Trauma Management (Sektion NIS) of the German Trauma Society. The participating hospitals submit their pseudonymized data to a central database via a web-based application. The participating hospitals are primarily located in Germany (90\%); however, an increasing number of foreign hospitals also contribute data, including hospitals from Austria, Belgium, China, Finland, Luxembourg, Slovenia, Switzerland, The Netherlands, and the United Arab Emirates. Currently, approximately 33,000 cases from over 650 hospitals are entered into the database per year. Participation in TR-DGU is voluntary. For hospitals associated with TraumaNetzwerk DGU ${ }^{\circledR}$, however, 
the entry of at least a basic data set is obligatory for reasons of quality assurance. The present study is in line with the publication guidelines of the TR-DGU, underwent an internal peer review and is registered as TR-DGU project ID 2019-028.

\section{Classifications}

\section{Injury severity assessment}

The Abbreviated Injury Scale (AIS) is an anatomical coding system for classifying and describing the severity of injuries in every body region, and it is used in many trauma registries. Based on the AIS values, the injury severity score (ISS) can be calculated to assess the cumulative trauma severity. Seriously injured is defined by an ISS $\geq 16$.

\section{Motor function assessment}

Neurological assessment was performed according to the Eppendorf-Cologne Scale (ECS) [21]. The ECS is a prognostic score for TBI that is based on the motor component and pupil response and size specified in the Glasgow Coma Scale (GCS). In our analysis, we exclusively used the motor response, which is classified as normal (0 points), specific (1 point), nonspecific ( 2 points), or completely absent ( 3 points). According to the literature, the ECS shows a significantly higher accuracy for the prediction of TBI presence compared to the GCS [22].

\section{Patient selection}

Patients aged 16 years or older who were treated primarily in a participating hospital between 2009 and 2016 were enrolled. Further inclusion and exclusion criteria are summarized in Fig. 1.

\section{Epidemiological analysis}

For the descriptive analysis of epidemiological data, we categorized spine injuries into three groups: none, AIS severity score of 2-3 (injuries with no or transient neurological sings), and AIS score of 4-6 (injuries with relevant neurological abnormalities). AIS scores of 1 were excluded because they are irrelevant to therapy or outcome in terms of severe injury care. For the regression analysis, an AIS 3-6 was chosen as a dependent variable because spine injuries classified as AIS $\geq 3$ are characterized as relevant injuries [23]. This is different to the descriptive presentation of the data. But for the predictors, the clinical relevance was important regarding the relevant spine injuries and thus immobilization, which will be necessary already for spinal fractures with an elevated risk of instability but without

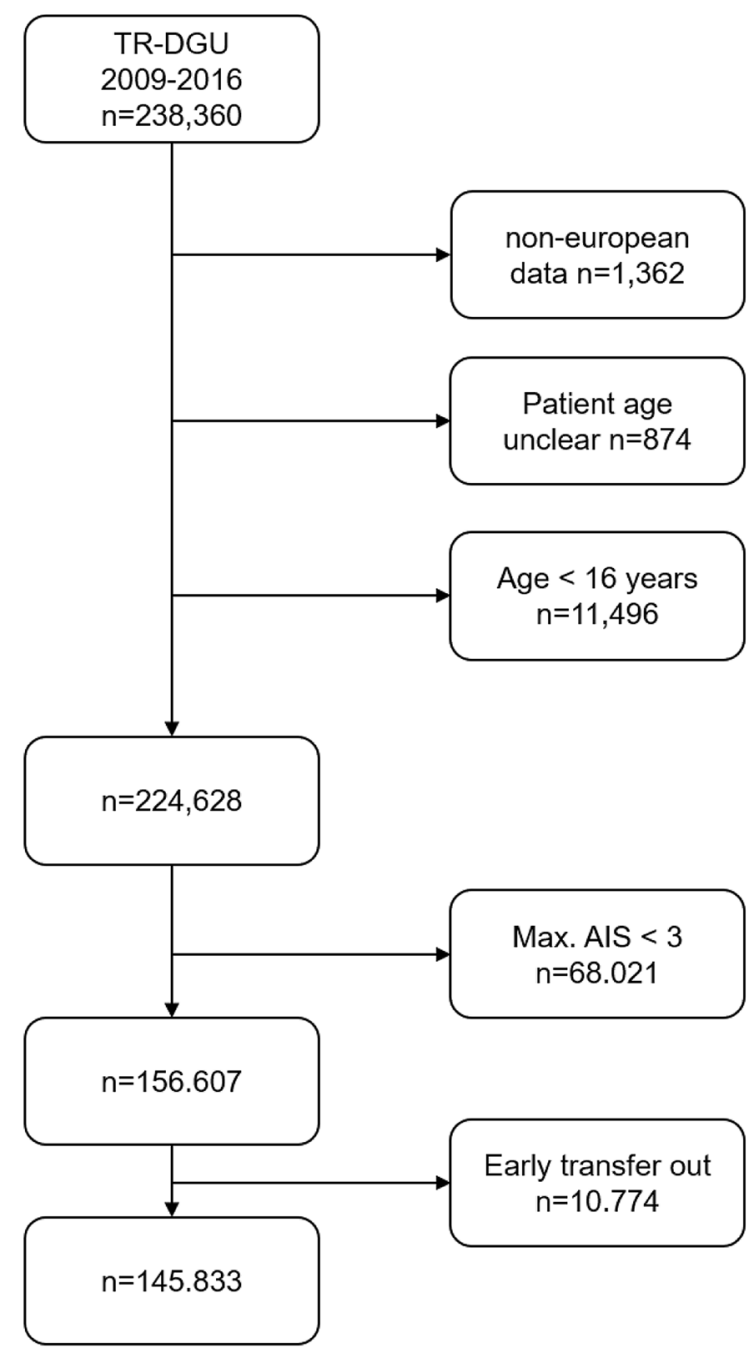

Fig. 1 Flowchart representing the inclusion and exclusion criteria

relevant neurological abnormalities, as well as for injuries of the spinal cord and other neural structures with neurological symptoms (AIS 3-6). AIS 2 injuries are often discovered later, are less relevant, and do not result in therapeutic decisions.

\section{Analysis of potential predictors}

To identify potential predictors that can predict severe spinal injuries (i.e., AIS $\geq 3$ ), a linear regression was performed with age, accident mechanism, accompanying injuries, vital signs, and motor response as potential predictors according to the ECS.

\section{Statistical analysis}

Data are presented as mean with standard deviation $( \pm \mathrm{SD})$ for continuous variables and as number of cases with 
percentages for categorical variables. $p$ values $<0.05$ were considered statistically significant. For the descriptive patient data, no $p$ values were given. Logistic regression was performed to identify predictors with a relevant impact on the main question. Regression coefficients are given with standard error and the respective $p$ value of the model, as well as odds ratio (OR) with $95 \%$ confidence interval (CI). Statistical analysis was performed using SPSS v.24.0 (IBM Inc., Armonk, NY, USA).

\section{Results}

During the study period from 2009 to 2016, 145,833 patients met the inclusion criteria (Fig. 1). The mean age was $52.7 \pm 21.1$ years, and $70.7 \%$ of the patients were male.
The hospital mortality rate was $13.9 \%(n=20,234)$, and the mean ISS was $21.8 \pm 11.8$.

\section{Epidemiological analysis}

Regarding the severity of spinal injuries, a total of 102,152 patients $(70.0 \%)$ had no spine injury, 37,749 patients (25.9\%) were classified as AIS 2-3, and 5932 patients $(4.1 \%)$ were classified as AIS 4-6 (Table 1). Among patients with spine injuries, cervical injuries were sustained by 11,095 (25.4\%) patients, the thoracic spine was injured in 17,517 (40.1\%) patients, and the lumbar spine was injured in 18,974 (43.4\%) patients (multiple choice). Spine injuries with AIS 4-6 were most common in patients between 60 and 79 years of age (Table 1); however, patients over the age of 80 years often sustained only minor spinal injuries. Thoracic and

Table 1 Demographic, injury, and vital parameter data of patients

\begin{tabular}{|c|c|c|c|c|}
\hline & No spinal injury & AIS 2-3 & AIS 4-6 & Overall patients \\
\hline Age & $n(\%)$ & $n(\%)$ & $n(\%)$ & $n(\%)$ \\
\hline $16-59$ years & $60,580(59.3 \%)$ & $24,260(64.3 \%)$ & $3701(62.4 \%)$ & $88,541(60.7 \%)$ \\
\hline $60-69$ years & $12,611(12.3 \%)$ & $4952(13.1 \%)$ & $812(13.7 \%)$ & $18,375(12.6 \%)$ \\
\hline $70-79$ years & $15,193(14.9 \%)$ & $5078(13.5 \%)$ & $886(14.9 \%)$ & $21,157(14.5 \%)$ \\
\hline$\geq 80$ years & $13,768(13.5 \%)$ & $3459(9.2 \%)$ & $533(9.0 \%)$ & $17,760(12.2 \%)$ \\
\hline Relevant injuries (AIS $\geq 3$ ) & $n(\%)$ & $n(\%)$ & $n(\%)$ & $n(\%)$ \\
\hline Head & $47,210(46.2 \%)$ & $12,584(33.3 \%)$ & $1228(20.7 \%)$ & $61,022(41.8 \%)$ \\
\hline Face & $5105(5.0 \%)$ & $1756(4.7 \%)$ & $135(2.3 \%)$ & $6996(4.8 \%)$ \\
\hline Thorax & $42,180(41.3 \%)$ & $22,814(60.4 \%)$ & $2383(40.2 \%)$ & $67,377(46.2 \%)$ \\
\hline Abdomen & $9172(9.0 \%)$ & $4144(11.0 \%)$ & $335(5.6 \%)$ & $13,651(9.4 \%)$ \\
\hline Extremities & $24,740(24.2 \%)$ & $7986(21.2 \%)$ & $545(9.2 \%)$ & $33,271(22.8 \%)$ \\
\hline Isolated TBI & $21,965(21.5 \%)$ & $1383(3.7 \%)$ & $1587(26.8 \%)$ & $24,935(17.1 \%)$ \\
\hline Multisystem trauma with TBI & $33,119(21.5 \% 9$ & $20,073(3.7 \%)$ & $2653(44.7 \%)$ & $55,845(38.3 \%)$ \\
\hline Vital signs & Mean (SD) & Mean (SD) & Mean (SD) & Mean (SD) \\
\hline GCS prehospital & $12.21(4.10)$ & $12.55(3.93)$ & $12.36(3.99)$ & $12.31(4.06)$ \\
\hline GCS pre-hospital, median & 14.00 & 15.00 & 14.00 & 14.00 \\
\hline SBP prehospital & $133.94(31.31)$ & $128.40(30.29)$ & $119.36(29.83)$ & $131.96(31.18)$ \\
\hline Heart frequency prehospital & $90.42(21.63)$ & $92.71(21.11)$ & $84.82(22.54)$ & 90.83 (21.19) \\
\hline SBP trauma room & $133.27(29.75)$ & $127.92(29.51)$ & $121.58(28.59)$ & $131.44(22.80)$ \\
\hline Heart frequency trauma room & $87.22(20.23)$ & $90.12(20.64)$ & $83.53(22.16)$ & $87.89(20.49)$ \\
\hline ECS motor response & $n(\%)$ & $n(\%)$ & $n(\%)$ & $n(\%)$ \\
\hline Normal & $59,083(64.9 \%)$ & $23,635(69.1 \%)$ & $3086(60.3 \%)$ & $85,804(65.8 \%)$ \\
\hline Specific & $16,643(18.3 \%)$ & $5519(16.1 \%)$ & $686(13.4 \%)$ & $22,848(17.5 \%)$ \\
\hline Unspecific & $3660(4.0 \%)$ & $1072(3.1 \%)$ & $120(2.3 \%)$ & $4852(3.7 \%)$ \\
\hline No response & $11,649(12.8 \%)$ & $3981(11.6 \%)$ & $1229(24.0 \%)$ & $16,859(12.9 \%)$ \\
\hline Minutes of patient care & Mean (SD) & Mean (SD) & Mean (SD) & Mean (SD) \\
\hline On-scene time $(n=38,955)$ & $28.4(50.9)$ & $29.9(16.8)$ & $30.15(16.3)$ & $28.9(16.5)$ \\
\hline Emergency department $(n=53,018)$ & $69.4(16.4)$ & $72.3(50.5)$ & $78.17(54.6)$ & $70.6(51.1)$ \\
\hline Hospital $\mathrm{SBP} \leq 90 \mathrm{mmHg}$ & $n(\%)$ & $n(\%)$ & $n(\%)$ & $n(\%)$ \\
\hline \multirow[t]{2}{*}{ Transfusion of packed red blood cells } & $8042(8.7 \%)$ & $4139(11.9 \%)$ & $895(16.9 \%)$ & $13,076(9.9 \%)$ \\
\hline & $3007(38.8 \%)$ & $1935(47.4 \%)$ & $306(34.9 \%)$ & $5248(41.3 \%)$ \\
\hline \multirow{2}{*}{$\begin{array}{l}\text { Diagnostic accuracy of pre-hospital emergency } \\
\text { doctors }\end{array}$} & $n(\%)$ & $n(\%)$ & $n(\%)$ & $n(\%)$ \\
\hline & $20,247(72.9 \%)$ & $4523(35.4 \%)$ & $332(13.2 \%)$ & $25,102(58.3 \%)$ \\
\hline Spine injury, none & $3068(11.0 \%)$ & $1587(12.4 \%)$ & $84(3.4 \%)$ & $4739(11.0 \%)$ \\
\hline Spine injury, slight & $3259(11.7 \%)$ & $4121(32.2 \%)$ & $366(14.6 \%)$ & $7746(18.0 \%)$ \\
\hline Spine injury, moderate & $1213(4.4 \%)$ & $2557(20.0 \%)$ & $1724(68.8 \%)$ & $5494(12.8 \%)$ \\
\hline Spine injury, serious & & & & \\
\hline
\end{tabular}

GCS Glasgow Coma Scale, SBP systolic blood pressure, TBI traumatic brain injury, ECS Eppendorf-Cologne Scale 
abdominal injuries were most prevalent in the AIS 2-3 group, while accompanying injuries to the head, face, and extremities were found mostly without injuries to the spine. Patients with TBI had an AIS 4-6 spinal injury score in $26.8 \%$ of cases. Spine injures was even more evident in patients with TBI who had combined multi-system trauma; here, $44.7 \%$ had spinal injuries with an AIS score of 4-6.

The vital signs, which are described in Table 1, show that the prehospital and trauma room mean blood pressure values were lowest in the AIS 4-6 group, whereas GCS and heart rate were highest in the AIS 2-3 group. The accuracy of the correct emergency-physician diagnosis of the spinal trauma was dependent on the severity of the injury. For this purpose, the severity of injury is subjectively assessed prehospital in categories that are compared to the final discharge diagnosis. While patients without spine injuries were overestimated in $27.1 \%$ of cases, patients with AIS 4-6 spinal injuries were underestimated in $31.2 \%$. The most considerable variation was found in the AIS 2-3 group, where $47.8 \%$ of the patients were underestimated and $20 \%$ were overestimated.

\section{Analysis of potential predictors}

Multivariate logistic regression analysis considered spine injury (AIS 3-6) with a prevalence of $10.6 \%$ and cervical spine injury (AIS 3-6) with a prevalence of 5.1\% as dependent variables. Blunt trauma was a remarkable independent variable for spinal trauma (OR 4.066, $p<0.001$ ) and cervical spine injury (OR 3.640, $p<0.001$ ). A fall from over $3 \mathrm{~m}$ was a predictor for a spine injury (OR 2.243, $p<0.001$ ), but not for cervical spine injury $(0.636, p<0.001)$. A pre-hospital GCS $\leq 8$ did not suggest a spinal injury per se, but a pre-hospital systolic blood pressure $\leq 90 \mathrm{mmHg}$ was predictive (spine OR $1.877, p<0.001$, and cervical spine OR 2.342, $p<0.001$ ). Patients aged 65 years and older were more likely to have cervical spine injuries. The diminished or absent motor response was a likely injury for both the spine and cervical spine (Table 2).

Table 2 Multivariate logistic regression analysis to predict any relevant spine injury (AIS 3-6, $n=15,481$; prevalence $=10.6 \%$ ), and relevant cervical spine injury (AIS 3-6, $n=5929$; prevalence $=5.1 \%$ )

\begin{tabular}{|c|c|c|c|c|c|c|c|c|}
\hline \multirow[t]{2}{*}{ Predictor } & \multicolumn{4}{|l|}{ Complete spine } & \multicolumn{4}{|l|}{ Cervical spine } \\
\hline & Coefficient (SE) & OR & $95 \% \mathrm{CI}$ & $p$ value & Coefficient (SE) & OR & $95 \% \mathrm{CI}$ & $p$ value \\
\hline Head AIS $\geq 3$ & $-1.655(0.032)$ & 0.191 & $0.180-0.203$ & $<0.001$ & $-1.700(0.047)$ & 0.183 & $0.166-0.200$ & $<0.001$ \\
\hline Face AIS $\geq 3$ & $-0.307(0.039)$ & 0.736 & $0.681-0.795$ & $<0.001$ & $-0.133(0.056)$ & 0.876 & $0.784-0.978$ & $=0.018$ \\
\hline Thorax AIS $\geq 3$ & $-0.996(0.024)$ & 0.369 & $0.352-0.387$ & $<0.001$ & $-1.418(0.040)$ & 0.242 & $0.224-0.262$ & $<0.001$ \\
\hline Abdomen AIS $\geq 3$ & $-0.838(0.043)$ & 0.432 & $0.397-0.471$ & $<0.001$ & $-1.131(0.077)$ & 0.323 & $0.277-0.375$ & $<0.001$ \\
\hline Extremities and pelvis AIS $\geq 3$ & $-1.421(0.032)$ & 0.241 & $0.227-0.257$ & $<0.001$ & $-1.679(0.056)$ & 0.186 & $0.167-0.208$ & $<0.001$ \\
\hline Male & $0.009(0.025)$ & 1.009 & $0.961-1.059$ & $=0.730$ & $0.210(0.039)$ & 1.234 & $1.144-1.332$ & $<0.001$ \\
\hline Blunt trauma & $1.403(0.092)$ & 4.066 & $3.395-4.870$ & $<0.001$ & $1.292(0.143)$ & 3.640 & $2.748-4.821$ & $<0.001$ \\
\hline \multicolumn{9}{|c|}{ Accident mechanism (reference car) } \\
\hline Motorcycle & $-0.007(0.038)$ & 0.993 & $0.922-1.070$ & $=0.852$ & $-0.323(0.063)$ & 0.724 & $0.640-0.818$ & $<0.001$ \\
\hline Bicycle & $-0.138(0.048)$ & 0.871 & $0.793-0.956$ & $=0.004$ & $-0.071(0.066)$ & 0.931 & $0.818-1.060$ & $=0.280$ \\
\hline Pedestrian & $-0.534(0.061)$ & 0.586 & $0.520-0.660$ & $<0.001$ & $-0.581(0.086)$ & 0.559 & $0.472-0.662$ & $<0.001$ \\
\hline Fall $>3 \mathrm{~m}$ & $0.808(0.031)$ & 2.243 & $2.109-2.385$ & $<0.001$ & $-0.453(0.056)$ & 0.636 & $0.570-0.710$ & $<0.001$ \\
\hline Fall $<3 \mathrm{~m}$ & $-0.050(0.037)$ & 0.951 & $0.885-1.022$ & $=0.172$ & $-0.163(0.053)$ & 0.850 & $0.765-0.943$ & $=0.002$ \\
\hline Other & $-0.152(0.045)$ & 0.859 & $0.787-0.939$ & $=0.001$ & $-0.467(0.070)$ & 0.627 & $0.547-0.718$ & $<0.001$ \\
\hline GCS $\leq 8$ pre-hospital & $-0.455(0.065)$ & 0.634 & $0.559-0.720$ & $<0.001$ & $-0.687(0.095)$ & 0.503 & $0.417-0.606$ & $<0.001$ \\
\hline $\mathrm{SBP} \leq 90 \mathrm{mmHg}$ & $0.630(0.032)$ & 1.877 & $1.763-1.999$ & $<0.001$ & $0.851(0.045)$ & 2.342 & $2.144-2.557$ & $<0.001$ \\
\hline \multicolumn{9}{|l|}{ Age } \\
\hline $65-79$ years & $-0.124(0.029)$ & 0.883 & $0.835-0.934$ & $<0.001$ & $0.295(0.043)$ & 1.344 & $1.236-1.461$ & $<0.001$ \\
\hline$\geq 80$ years & $-0.334(0.042)$ & 0.716 & $0.660-0.777$ & $<0.001$ & $0.275(0.056)$ & 1.316 & $1.179-1.470$ & $<0.001$ \\
\hline \multicolumn{9}{|l|}{ ECS motor response } \\
\hline Specific & $0.153(0.033)$ & 1.165 & $1.091-1.244$ & $<0.001$ & $0.311(0.052)$ & 1.365 & $1.232-1.513$ & $<0.001$ \\
\hline Unspecific & $0.431(0.088)$ & 1.539 & $1.296-1.827$ & $<0.001$ & $0.870(0.127)$ & 2.388 & $1.862-3.061$ & $<0.001$ \\
\hline No response & $1.154(0.069)$ & 3.171 & $2.768-3.633$ & $<0.001$ & $2.010(0.098)$ & 7.462 & $6.160-9.039$ & $<0.001$ \\
\hline
\end{tabular}

ECS Eppendorf-Cologne Scale 


\section{Discussion}

Our data describe a prevalence of $10.6 \%$ for relevant spinal trauma and $5.1 \%$ for severe cervical spine trauma in severely injured trauma patients in Europe. The prevalence of severe cervical spinal injuries described in our study is comparable to that found in studies of other countries $[15,17,24]$. Regarding potential predictors, independent variables were evaluated using regression analysis, which might yield differences compared to other calculations given the categories and characteristics used.

Blunt trauma was found to be predictive for a spinal injury and cervical spinal injury. Severe spine injury is four times more likely to result from a blunt trauma than a penetrating trauma. In line with the literature, this implies that patients with penetrating trauma should not be immobilized because spinal injury is unlikely and immobilization could delay lifesaving interventions [10, 25-28].

As expected, motor deficits in the clinical examination were an outstanding predictor for spine injuries, particularly cervical spine injuries. A lowered level of consciousness is often mentioned as a predictor of spinal trauma; however, in our regression analysis, a pre-hospital GCS $<9$ was not a predictor for spinal injury. Our results corroborate those in the literature that, with severe isolated TBI or with TBI in combination with other injuries, severe injuries to the spine can be expected $[29,30]$.

Accident mechanisms are expected to provide an idea of the possible injuries, and discussions on this topic remain controversial [27, 31]. In our analysis, the documented accident mechanisms correlate poorly with cervical spinal injuries, although other studies have found otherwise [30]. Thus, we contend that an accident mechanism can only be considered as a risk factor that has to lead health professionals to an increased attention for cervical spinal injuries. However, falls from a height of more than $3 \mathrm{~m}$ were a precise predictor for an injury to the entire spine, which had already been confirmed by other studies $[15,27]$.

Patients aged 65 years and older were found to be more frequently affected by cervical spine injuries. The literature describes low-energy injuries, low-risk mechanisms, decreases in total mobility, and degenerative changes as causative factors [32, 33]. Additionally, a blood pressure $\leq 90 \mathrm{mmHg}$ was found to be a significant predictor for the spine and cervical spine injuries. It was initially unclear whether hypotension is due to a neuronal disorder or hemorrhage. Table 1 shows that $41.3 \%$ of patients with a systolic blood pressure (SBP) $\leq 90 \mathrm{mmHg}$ received blood transfusions in the shock room. Compared to the other characteristics, this is perhaps the most likely indication of hemorrhage in the context of overall injury severity.
That thoracic and abdominal injuries were most prevalent in the AIS 2-3 group confirms our expectations. This should be kept in mind for spinal injury, especially with increasing trauma severity and the number of accompanying injuries. Multiply injured patients in combination with TBI, as well as an expression of severely injured patients, are also more likely to have severe spinal cord injuries (AIS 4-6). Therefore, hemodynamic instability should be interpreted as a sign of high overall injury severity, which affects the spine in general and the cervical spine in particular. The fact that nearly $50 \%$ of the diagnostic certainty is faulty is regularly described in the literature and underlines the need for a simple but easy-to-use decision aid for (prehospital) immobilization [29, 34].

Currently, there are a mere handful of recommendations proposed for spinal immobilization $[10,11,35]$. These recommendations often consider the accident mechanism and abnormalities in the clinical examination equivalent, which failed to be confirmed by our analysis. Also, the German polytrauma guideline gives greater weight to clinical findings [27]. It is indisputable that life-threatening findings should take priority over immobilization measures $[10,11$, 35].

Though pain is not documented in the TR-DGU, it would seem logical to take pain under consideration from a clinical standpoint. By way of example, a retrospective study has shown that immobilization was performed in $37.2 \%$ of patients due to back pain [36]. A cut-off for pain was not described, so that "the worse the pain, the more likely is immobilization" can be noted first, but immobilization is required, at the latest, when the patient requires analgesia. Healthcare professionals must be trained to interpret pain as a warning signal, but also to administer adequate analgesics [37]. Ultimately, however, as with every suspicion of benign fracture, a decision on whether splinting or immobilization is most appropriate must be made.

In case of an inconspicuous clinical examination and the presence of risk factors, immobilization does not seem to be superior to independent, pain-free relieving posture, although this does not preclude spinal injury [38-41]. Also, data show that the physical examination for possible spinal cord injury is not affected by the injury mechanism [31]. This raises the question of whether, due to the disadvantages of immobilization (e.g., discomfort, pressure points, restrictions of ventilation), "spinal motion restriction" exclusively is considered sufficient to prevent large, uncontrolled, passive movements (e.g., by transport) without securing the patient to a mattress or board.

For immobilization in TBI, it is important to know, that there are studies with minimal numbers of cases, some of which are almost 20 years old, which describe an increase in intracranial pressure (ICP) or increased internal jugular vein cross-sectional area when using a cervical collar [42-48]. 
A current meta-analysis with only 86 patients showed a significant overall increase in ICP after collar application (weighted mean difference $=4.43 ; 95 \%$ CI $1.70-7.17$; $p<0.01$ ) [49]. In this respect, the use of a cervical collar should certainly be avoided, and the cervical spine should be immobilized by other measures (manual, head blocks, etc.).

The much-described upper-body elevation (or head-ofbed elevation) in TBI has only weak evidence. In a Cochrane review on head-of-bed elevation in clinical care, no clear recommendation could be given [50]. In this respect, it must be considered for the acute phase whether an upperbody elevation, e.g., if isolated TBI is sensible, or whether additional injuries, physiological parameters of the rescuespecific aspects speak against it.

The best form of immobilization for transport (e.g., vacuum mattress, spineboard, stretcher) remains unclear despite numerous qualitatively heterogeneous, well-performed, and clinically relevant studies. How a patient must be immobilized or transported with low mobility remains a topic of active discussion. Some studies, for example, prefer the vacuum mattress for immobilization [51, 52]. Aside from the comfort of lying down, a recent exploratory biomechanical study found that the spineboard, followed by the stretcher with head immobilization, immobilizes better than the vacuum mattress [3]. Uzun et al. show that the remaining movement of the cervical spine is minimal when the patient is immobilized on a spine board with a headlock system or vacuum mattress with additional head blocks. The remaining movement of the cervical spine could not be reduced by the additional use of a cervical collar.[53]. So it seems to be superfluous. But it should be noted, however, that some paramedics-especially emergency physicians-lack proper immobilization skills [54]. Regular skills training, as well as risk assessment skills, are required to achieve improvements $[55,56]$.

It appears essential to keep in mind that immobilization does not influence neurological trauma; rather, it is merely intended to prevent further damage throughout the extrication and transport processes [6, 41, 57]. In addition to the clinical symptoms of pain, we see spill (fall $>3 \mathrm{~m}$ ), seniority (age $>65$ years), seriously injured, and skull/traumatic brain injury, which we term the " $4 \mathrm{~S}$ ", as an indication for increased attention by health professionals for cervical spinal injuries or indication for spinal motion restriction. The " $4 \mathrm{~S}$ " could prove useful as a memory aid, similar to the " $3 \mathrm{~S}$ " (scene, safety, situation) from emergency training for assessing a site.

As with all retrospective analyses, the present study has limitations. The trauma register does not include patients who died at the site of the accident; it only includes patients who reached the hospital alive. Given that we aimed to analyze severely injured persons, the applicability of our conclusions to patients who were less severely injured is questionable. We decided to include spine injuries from AIS $\geq 3$ (serious injury $=$ unstable fractures and neurological deficits). Stable fractures (AIS 2) are often diagnosed late and may, therefore, be less important for acute care during the initial stages of treatment. However, the AIS score estimates the severity of the injury rather than the impact of energy input, and the accident mechanism can only be differentiated rudimentarily because detailed information in the TR-DGU is not recorded.

\section{Conclusion}

In summary, the results of this registry analysis should be included in the assessment of severely injured patients for the indication of spinal immobilization. For clinical evaluation, symptoms are more significant than kinematics. The risk factors (4S: spills, seniority, seriously injured, skull trauma) should focus the examination on the spine. If an examination is not possible because of impaired vigilance, the spine should be immobilized in case of doubt if the trauma mechanism is relevant. For logical considerations, in case of a vital risk, immobilization should not delay therapy or transport. Spinal injuries from isolated penetrating trauma should not be immobilized.

Author contributions DH, MK and RL conceived the study and DH and RL designed the trial. RL and DH did the statistical analysis RL supervised statistics. Data interpretation was done by RL, DH, JPS. DH and JPS drafted the article, a critical revision of the article was done by MK, the final approval of the version to be published was done by all authors.

Funding Open Access funding enabled and organized by Projekt DEAL.

Code availability Not applicable.

\section{Compliance with ethical standards}

Conflict of interest The authors declare that they have no competing interests.

Ethics approval and consent to participate The present study is in line with the publication guidelines of the TR-DGU, underwent an internal peer review and is registered as TR-DGU project ID 2019-028.

Availability of data and material (data transparency) Data are provided by the TraumaRegister $D U^{\circledR}$. Data are available from the TraumaRegister $\mathrm{DGU}^{\circledR}$ for researchers who meet the criteria for access to confidential data.

Open Access This article is licensed under a Creative Commons Attribution 4.0 International License, which permits use, sharing, adaptation, distribution and reproduction in any medium or format, as long as you give appropriate credit to the original author(s) and the source, provide a link to the Creative Commons licence, and indicate if changes 
were made. The images or other third party material in this article are included in the article's Creative Commons licence, unless indicated otherwise in a credit line to the material. If material is not included in the article's Creative Commons licence and your intended use is not permitted by statutory regulation or exceeds the permitted use, you will need to obtain permission directly from the copyright holder. To view a copy of this licence, visit http://creativecommons.org/licenses/by/4.0/.

\section{References}

1. Kreinest M, Gliwitzky B, Goller S, Münzberg M. Präklinische Immobilisation der Wirbelsäule. Notfall Rettungsmed. 2016;19:41-7. https://doi.org/10.1007/s10049-015-0118-5.

2. Sundstrøm T, Asbjørnsen H, Habiba S, Sunde GA, Wester K. Prehospital use of cervical collars in trauma patients: a critical review. J Neurotrauma. 2014;31:531-40. https://doi.org/10. 1089/neu.2013.3094.

3. Nolte PC, Uzun DD, Häske D, Weerts J, Münzberg M, Rittmann A, et al. Analysis of cervical spine immobilization during patient transport in emergency medical services. Eur J Trauma Emerg Surg. 2019;26:S2. https://doi.org/10.1007/ s00068-019-01143-z.

4. Kreinest M, Ludes L, Türk A, Grützner PA, Biglari B, Matschke $\mathrm{S}$. Analysis of prehospital care and emergency room treatment of patients with acute traumatic spinal cord injury: a retrospective cohort study on the implementation of current guidelines. Spinal Cord. 2017;55:16-9. https://doi.org/10.1038/sc.2016.84.

5. Kwan I, Bunn F, Roberts I. Spinal immobilisation for trauma patients. Cochrane Database Syst Rev. 2001. https://doi.org/10. 1002/14651858.CD002803.

6. Hauswald M, Ong G, Tandberg D, Omar Z. Out-of-hospital spinal immobilization: its effect on neurologic injury. Acad Emerg Med. 1998;5:214-9. https://doi.org/10.1111/j.1553-2712.1998.tb026 15.x.

7. Hood N, Considine J. Spinal immobilisaton in pre-hospital and emergency care: a systematic review of the literature. Australas Emerg Nurs J. 2015;18:118-37. https://doi.org/10.1016/j.aenj. 2015.03.003.

8. Liao S, Schneider NRE, Hüttlin P, Grützner PA, Weilbacher F, Matschke $S$, et al. Motion and dural sac compression in the upper cervical spine during the application of a cervical collar in case of unstable craniocervical junction-a study in two new cadaveric trauma models. PLoS ONE. 2018;13:e0195215. https://doi.org/10. 1371/journal.pone.0195215.

9. Ham W, Schoonhoven L, Schuurmans MJ, Leenen LPH. Pressure ulcers from spinal immobilization in trauma patients: a systematic review. J Trauma Acute Care Surg. 2014;76:1131-41. https://doi. org/10.1097/TA.0000000000000153.

10. Kreinest M, Gliwitzky B, Schüler S, Grützner PA, Münzberg M. Development of a new emergency medicine spinal immobilization protocol for trauma patients and a test of applicability by German emergency care providers. Scand J Trauma Resusc Emerg Med. 2016;24:71. https://doi.org/10.1186/s13049-016-0267-7.

11. Maschmann C, Jeppesen E, Rubin MA, Barfod C. New clinical guidelines on the spinal stabilisation of adult trauma patientsconsensus and evidence based. Scand J Trauma Resusc Emerg Med. 2019;27:1-10. https://doi.org/10.1186/s13049-019-0655-x.

12. Stiell IG, Wells GA, Vandemheen KL, Clement CM, Lesiuk H, de Maio VJ, et al. The Canadian C-spine rule for radiography in alert and stable trauma patients. JAMA. 2001;286:1841-8. https://doi. org/10.1001/jama.286.15.1841

13. Hoffman JR, Wolfson AB, Todd K, Mower WR. Selective cervical spine radiography in blunt trauma: methodology of the National Emergency X-Radiography Utilization Study (NEXUS). Ann
Emerg Med. 1998;32:461-9. https://doi.org/10.1016/S01960644(98)70176-3.

14. Hanson JA, Blackmore CC, Mann FA, Wilson AJ. Cervical spine injury: a clinical decision rule to identify high-risk patients for helical CT screening. AJR Am J Roentgenol. 2000;174:713-7. https://doi.org/10.2214/ajr.174.3.1740713.

15. Hasler RM, Exadaktylos AK, Bouamra O, Benneker LM, Clancy M, Sieber R, et al. Epidemiology and predictors of spinal injury in adult major trauma patients: European cohort study. Eur Spine J. 2011;20:2174-80. https://doi.org/10.1007/s00586-011-1866-7.

16. Hasler RM, Exadaktylos AK, Bouamra O, Benneker LM, Clancy $\mathrm{M}$, Sieber R, et al. Epidemiology and predictors of cervical spine injury in adult major trauma patients: a multicenter cohort study. J Trauma Acute Care Surg. 2012;72:975-81. https://doi.org/10. 1097/TA.0b013e31823f5e8e.

17. Stephan K, Huber S, Häberle S, Kanz K-G, Bühren V, van Griensven M, et al. Spinal cord injury-incidence, prognosis, and outcome: an analysis of the TraumaRegister DGU. Spine J. 2015;15:1994-2001. https://doi.org/10.1016/j.spinee.2015.04. 041.

18. Brand S, Schmucker U, Lob G, Haasper C, Juhra C, Hell W, et al. Warum wieder mehr Menschen im Straßenverkehr sterbenTrendwende oder Ausreißer? Zentralbl Chir. 2017;142:209-15. https://doi.org/10.1055/s-0033-1350867.

19. Begley J. Car safety wars: one hundred years of technology, politics, and death. Bus Hist. 2019;74:1-3. https://doi.org/10.1080/ 00076791.2019.1651968.

20. Vaillancourt C, Stiell IG, Beaudoin T, Maloney J, Anton AR, Bradford $\mathrm{P}$, et al. The out-of-hospital validation of the Canadian C-spine rule by paramedics. Ann Emerg Med. 2009;54(663671):e1. https://doi.org/10.1016/j.annemergmed.2009.03.008.

21. Hoffmann M, Lehmann W, Rueger JM, Lefering R. Introduction of a novel trauma score. J Trauma Acute Care Surg. 2012;73:160713. https://doi.org/10.1097/TA.0b013e318270d572.

22. Hoffmann M, Lehmann W, Schroeder M, Cramer C, Grossterlinden LG, Rueger JM, Lefering R. Prospective evaluation of the Eppendorf-Cologne Scale. Eur J Emerg Med. 2017;24:120-5. https://doi.org/10.1097/MEJ.0000000000000306.

23. TRAUMA.ORG. Abbreviated Injury Scale. 03.02.2007. https:// www.trauma.org/archive/scores/ais.html. Accessed 3 May 2019.

24. Chiu WC, Haan JM, Cushing BM, Kramer ME, Scalea TM. Ligamentous injuries of the cervical spine in unreliable blunt trauma patients: incidence, evaluation, and outcome. J Trauma. 2001;50:457-63. https://doi.org/10.1097/00005373-20010300000009 (discussion 464).

25. Connell RA, Graham CA, Munro PT. Is spinal immobilisation necessary for all patients sustaining isolated penetrating trauma? Injury. 2003;34:912-4. https://doi.org/10.1016/S0020-1383(03) 00070-6.

26. Haut ER, Kalish BT, Efron DT, Haider AH, Stevens KA, Kieninger AN, et al. Spine immobilization in penetrating trauma: more harm than good? J Trauma. 2010;68:115-20. https://doi. org/10.1097/TA.0b013e3181c9ee58 (discussion 120-1).

27. Polytrauma Guideline Update Group. Level 3 guideline on the treatment of patients with severe/multiple injuries: AWMF Register-Nr. 012/019: AWMF Register-Nr. 012/019. Eur J Trauma Emerg Surg. 2018;44:3-271. https://doi.org/10.1007/ s00068-018-0922-y.

28. NAEMT. Präklinisches Traumamanagement: Prehospital trauma life support (PHTLS). 3rd ed. München: Elsevier Urban \& Fischer; 2016.

29. Schinkel C, Frangen TM, Kmetic A, Andress H-J, Muhr G. Wirbelsäulenfrakturen bei Mehrfachverletzten : Eine Analyse des DGU-Traumaregisters. Unfallchirurg. 2007;110:946-52. https:// doi.org/10.1007/s00113-007-1351-2. 
30. Tian H-L, Guo Y, Hu J, Rong B-Y, Wang G, Gao W-W, et al. Clinical characterization of comatose patients with cervical spine injury and traumatic brain injury. J Trauma. 2009;67:1305-10. https://doi.org/10.1097/TA.0b013e31819db57c.

31. Domeier RM, Evans RW, Swor RA, Hancock JB, Fales W, Krohmer J, et al. The reliability of prehospital clinical evaluation for potential spinal injury is not affected by the mechanism of injury. Prehosp Emerg Care. 1999;3:332-7. https://doi.org/10. 1080/10903129908958964.

32. Squarza S, Uggetti CL, Politi MA, Pescatori LC, Bisogno R, Campi A, et al. C1-C2 fractures in asymptomatic elderly patients with minor head trauma: evaluation with a dedicated head CT protocol. Radiol Bras. 2019;52:17-23. https://doi.org/10.1590/ 0100-3984.2017.0154.

33. Jeanmonod R, Varacallo M. Geriatric cervical spine injury. Treasure Island: StatPearls Publishing; 2019.

34. Helm M, Faul M, Unger T, Lampl L. Reliability of emergency medical field triage: exemplified by traffic accident victims. Anaesthesist. 2013;62:973-80. https://doi.org/10.1007/ s00101-013-2255-x.

35. Kornhall DK, Jørgensen JJ, Brommeland T, Hyldmo PK, Asbjørnsen H, Dolven T, et al. The Norwegian guidelines for the prehospital management of adult trauma patients with potential spinal injury. Scand J Trauma Resusc Emerg Med. 2017;25:2. https://doi.org/10.1186/s13049-016-0345-x.

36. Oosterwold JT, Sagel DC, van Grunsven PM, Holla M, de Man-van Ginkel J, Berben S. The characteristics and pre-hospital management of blunt trauma patients with suspected spinal column injuries: a retrospective observational study. Eur J Trauma Emerg Surg. 2017;43:513-24. https://doi.org/10.1007/ s00068-016-0688-z.

37. Häske D, Böttiger BW, Bouillon B, Fischer M, Gaier G, Gliwitzky $\mathrm{B}$, et al. Analgesia in patients with trauma in emergency medicine: a systematic review and meta-analysis. Dtsch Arztebl Int. 2017;114:785-92. https://doi.org/10.3238/arztebl.2017.0785.

38. Benger J, Blackham J. Why do we put cervical collars on conscious trauma patients? Scand J Trauma Resusc Emerg Med. 2009;17:1-4. https://doi.org/10.1186/1757-7241-17-44.

39. Rogers L. No place for the rigid cervical collar in pre-hospital care. Int Paramed Pract. 2017;7:12-5. https://doi.org/10.12968/ ippr.2017.7.1.12.

40. Stuke LE, Pons PT, Guy JS, Chapleau WP, Butler FK, McSwain NE. Prehospital spine immobilization for penetrating traumareview and recommendations from the Prehospital Trauma Life Support Executive Committee. J Trauma. 2011;71:763-9. https:// doi.org/10.1097/TA.0b013e3182255cb9 (discussion 769-70).

41. Tatum JM, Melo N, Ko A, Dhillon NK, Smith EJT, Yim DA, et al. Validation of a field spinal motion restriction protocol in a level I trauma center. J Surg Res. 2017;211:223-7. https://doi.org/10. 1016/j.jss.2016.12.030.

42. Craig GR, Nielsen MS. Rigid cervical collars and intracranial pressure. Intensive Care Med. 1991;17:504-5.

43. Davies G, Deakin C, Wilson A. The effect of a rigid collar on intracranial pressure. Injury. 1996;27:647-9. https://doi.org/10. 1016/S0020-1383(96)00115-5.

44. Hunt K, Hallworth S, Smith M. The effects of rigid collar placement on intracranial and cerebral perfusion pressures.
Anaesthesia. 2001;56:511-3. https://doi.org/10.1046/j.1365-2044. 2001.02053.x.

45. Kolb JC, Summers RL, Galli RL. Cervical collar-induced changes in intracranial pressure. Am J Emerg Med. 1999;17:135-7. https:// doi.org/10.1016/S0735-6757(99)90044-X.

46. Raphael JH, Chotai R. Effects of the cervical collar on cerebrospinal fluid pressure. Anaesthesia. 1994;49:437-9. https://doi.org/ 10.1111/j.1365-2044.1994.tb03482.x.

47. Mobbs RJ, Stoodley MA, Fuller J. Effect of cervical hard collar on intracranial pressure after head injury. ANZ J Surg. 2002;72:389_ 91. https://doi.org/10.1046/j.1445-2197.2002.02462.x.

48. Stone MB, Tubridy CM, Curran R. The effect of rigid cervical collars on internal jugular vein dimensions. Acad Emerg Med. 2010;17:100-2. https://doi.org/10.1111/j.1553-2712.2009. 00624.x.

49. Núñez-Patiño RA, Rubiano AM, Godoy DA. Impact of cervical collars on intracranial pressure values in traumatic brain injury: a systematic review and meta-analysis of prospective studies. Neurocrit Care. 2020;32:469-77. https://doi.org/10.1007/ s12028-019-00760-1.

50. Alarcon JD, Rubiano AM, Okonkwo DO, Alarcón J, MartinezZapata MJ, Urrútia G, Bonfill CX. Elevation of the head during intensive care management in people with severe traumatic brain injury. Cochrane Database Syst Rev. 2017;12:CD009986. https:// doi.org/10.1002/14651858.CD009986.pub2.

51. Wampler DA, Pineda C, Polk J, Kidd E, Leboeuf D, Flores M, et al. The long spine board does not reduce lateral motion during transport - a randomized healthy volunteer crossover trial. Am J Emerg Med. 2016;34:717-21. https://doi.org/10.1016/j.ajem. 2015.12.078.

52. Johnson DR, Hauswald M, Stockhoff C. Comparison of a vacuum splint device to a rigid backboard for spinal immobilization. Am J Emerg Med. 1996;14:369-72. https://doi.org/10.1016/S07356757(96)90051-0.

53. Uzun DD, Jung MK, Weerts J, Münzberg M, Grützner PA, Häske D, Kreinest M. Remaining cervical spine movement under different immobilization techniques. Prehosp Disaster Med. 2020;35:382-7. https://doi.org/10.1017/S1049023X2000059X.

54. Kreinest M, Goller S, Rauch G, Gliwitzky B, Frank C, Matschke $\mathrm{S}$, et al. Einflussfaktoren auf die präklinische Anlage einer Zervikalstütze. Unfallchirurg. 2017;120:675-82. https://doi.org/10. 1007/s00113-016-0207-z.

55. Kreinest M, Goller S, Gliwitzky B, Grützner PA, Küffer M, Häske $\mathrm{D}$, et al. Expertise of German paramedics concerning the prehospital treatment of patients with spinal trauma. Eur J Trauma Emerg Surg. 2017;43:371-6. https://doi.org/10.1007/s00068-016-0682-5.

56. Häske D, Dorau W, Heinemann N, Stock J-P, Schempf B. Development of an adapted model for decision-making to improve reasoning and risk assessment in an emergency team: A Prospective Simulation Study. Medicina (Kaunas). 2019;55:339. https://doi. org/10.3390/medicina55070339.

57. Fischer PE, Perina DG, Delbridge TR, Fallat ME, Salomone JP, Dodd J, et al. Spinal motion restriction in the trauma patient-a joint position statement. Prehosp Emerg Care. 2018;22:659-61. https://doi.org/10.1080/10903127.2018.1481476. 